\title{
Iris extramedullary hematopoiesis in choroidal melanoma
}

\author{
Beatrice Gallo ${ }^{1,2}$, Rana'a T. Al-Jamal ${ }^{3}$, Caroline Thaung ${ }^{4}$, Victoria M. L. Cohen ${ }^{1,2,4,5}$
}

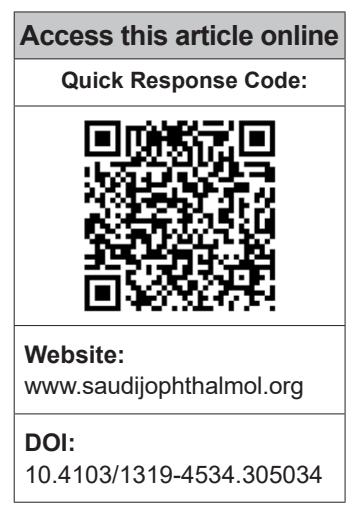

${ }^{1}$ Ocular Oncology Service, Moorfields Eye Hospital, ${ }^{2}$ Ocular Oncology Service, St Bartholomew's Hospital, ${ }^{3}$ Ocular Oncology Service, Helsinki University Central Hospital, Helsinki, Finland, ${ }^{4}$ Institute of Ophthalmology, University College London, ${ }^{5} \mathrm{NIHR}$ Biomedical Research Centre for Ophthalmology at Moorfields Eye Hospital and University College London, Institute of Ophthalmology, London, UK

Address for correspondence: Dr. Victoria M. L. Cohen, Ocular Oncology Service, Moorfields Eye Hospital, London EC1V 2PD, UK. E-mail: victoria.cohen@nhs.

Submitted: 10-Jun-2018 Revised: 09-Aug-2018 Accepted: 29-May-2019 Published: 28-Dec-2020

\section{Abstract:}

PURPOSE: Extramedullary hematopoiesis (EMH) usually occurs in patients with loss of bone marrow hematopoietic function, and in a vast majority of cases, it involves the liver, the spleen, or the lymph nodes. We report EMH in the iris of patients enucleated for choroidal melanoma $(\mathrm{CM})$.

METHODS: We report a series of three patients with CM, two treated with primary enucleation and one with secondary enucleation.

RESULTS: Histopathology revealed EMH in the iris of all patients.

CONCLUSION: EMH of the iris can be associated with CM in the absence of any hematological or systemic disorders. To the best of our knowledge, our work is the first report of this abnormal histopathological finding. Keywords:

Choroidal melanoma, enucleation, iris extramedullary hematopoiesis

\section{INTRODUCTION}

$\mathrm{U}$ veal melanoma (UM) is the most common adult primary intraocular malignancy. The estimated incidence varies from 2 to 8 new cases per million per year, depending on the latitude, and it is more frequently observed in fair skinned subjects; ${ }^{[1,2]}$ the mean age at diagnosis is 62 years. ${ }^{[3]}$ It arises from the melanocytes of the choroid in $90 \%$ of the cases, ciliary body in $6 \%$, and iris in $4 \%{ }^{[4]}$

Choroidal melanoma $(\mathrm{CM})$ can present in three different patterns: dome-shaped (75\%), mushroom-shaped (20\%), and diffuse $(5 \%){ }^{[5]}$ Approximately $50 \%$ of the patients with $\mathrm{CM}$ die from metastatic disease within 15 years, with the liver being the most common metastatic site. ${ }^{[6]}$ The mean survival after metastatic disease is diagnosed is 6-12 months. ${ }^{[7]}$ Histopathologically, $\mathrm{CM}$ is divided into spindle, epithelioid, and mixed cell types. Spindle type carries the best prognosis, while epithelioid type carries the worst. ${ }^{[4]}$

Extramedullary hematopoiesis (EMH) is a pathological term used to describe a compensatory

This is an open access journal, and articles are distributed under the terms of the Creative Commons Attribution-NonCommercial-ShareAlike 4.0 License, which allows others to remix, tweak, and build upon the work non-commercially, as long as appropriate credit is given and the new creations are licensed under the identical terms.

For reprints contact: WKHLRPMedknow_reprints@wolterskluwer.com reaction occurring in hematological disorders (thalassemia, myelofibrosis, polycythemia vera, and chronic myeloid leukemia) when there is an insufficient production of blood elements in the marrow of long bones, ribs, and vertebrae.$^{[8]}$ Liver, spleen, and lymph nodes are typical sites of EMH. ${ }^{[9]}$ The exact mechanism of this phenomenon is not well understood. Hill and Swanson observed EMH in nonhematological diseases such as myocardial infarction and hypothesized that it could be related to inflammation or tissue repair-associated trophic factors. ${ }^{[10]}$ Wolf and Neiman suggested that, in these disorders, EMH could be related to an abnormal release in the bloodstream of hematological precursors caused by an altered marrow architecture. ${ }^{[11]}$ Herein, we report three cases of large CM, two treated with primary enucleation and one with secondary enucleation, whose histopathological results showed EMH in the iris. To the best of our knowledge, this association has not been previously described.

\section{Design and Case Series}

Eligible for this study were three patients who had the globe enucleated for CM or ciliary body melanoma at Moorfields Eye Hospital, London, during a period of

How to cite this article: Gallo B, Al-Jamal RT, Thaung C, Cohen VM. Iris extramedullary hematopoiesis in choroidal melanoma. Saudi J Ophthalmol 2020;34:82-4. 
5 years (2013-2018). The study adhered to the tenets of the Declaration of Helsinki.

The first patient was a 66-year-old man with unremarkable general health, having a history of right eye gradual visual deterioration, and on dilated fundus examination presenting an inferior exudative retinal detachment which extended up to the inferior vascular arcades. The B-scan ultrasound showed an elevated diffuse sessile lesion of $2.7 \mathrm{~mm}$ in thickness and $23 \mathrm{~mm}$ in longitudinal base, with low-to-medium internal echogenicity. Due to the extensive retinal detachment and the poor visualization of the lesion, diffuse $\mathrm{CM}$, intraocular lymphoma, and choroidal metastasis were included in the differential diagnosis. The systemic screening denied any primary malignancies elsewhere or metastatic deposits from the primary intraocular tumor; therefore, vitrectomy with choroidal biopsy and injection of silicone oil was performed. The histopathological examination confirmed the diagnosis of $\mathrm{CM}$, and due to the large tumor size, the patient underwent primary enucleation. The pathological examination of the enucleated globe disclosed extramedullary hematopoiesis within the iris [Figure 1a]. The iris on clinical examination before surgery was described as normal.

The second patient was a 56-year-old male in good general health and with a family history for $\mathrm{CM}$; he presented with a visual field defect in the right eye and fundal examination revealed a choroidal mass nasal to the optic disc with associated exudative retinal detachment; the B-scan ultrasound showed that the lesion had a thickness of $5.2 \mathrm{~mm}$ and low-to-medium internal reflectivity. The tumor was treated with Ruthenium plaque brachytherapy followed by two sessions of diode laser thermotherapy; unfortunately, 5 years later, the patient developed recurrent vitreous hemorrhages that rendered necessary vitrectomy, later complicated by intractable neovascular glaucoma and hyphema, which led to secondary enucleation. The pathology disclosed spindle cell-type CM and the iris showed focal EMH [Figure 1b and c].

The third patient was a 75-year-old male referred to our service for an unexplained vitreous hemorrhage. Initially, he underwent vitrectomy and injection of silicone oil followed by silicone oil and cataract removal that was subsequently complicated by hyphema with blood dispersion in the anterior chamber precluding iris and fundal view; the B-scan ultrasound detected a lesion of $5.1 \mathrm{~mm}$ in elevation and $12.4 \mathrm{~mm}$ and $12.1 \mathrm{~mm}$ of longitudinal and transverse base, with low-to-medium internal echogenicity and intrinsic blood flow highly suggestive of $\mathrm{CM}$. Three months after, the patient underwent enucleation as the absent view of the tumor did not allow any globe-saving treatments. The histopathological examination confirmed the diagnosis of spindle cell-type CM and EMH in the iris stroma [Figure 1d].

In all three cases, no vortex vein invasion or extrascleral extension was observed. In all patients, at the time of diagnosis, the systemic investigations denied primary malignancies elsewhere or metastatic deposits from the primary intraocular

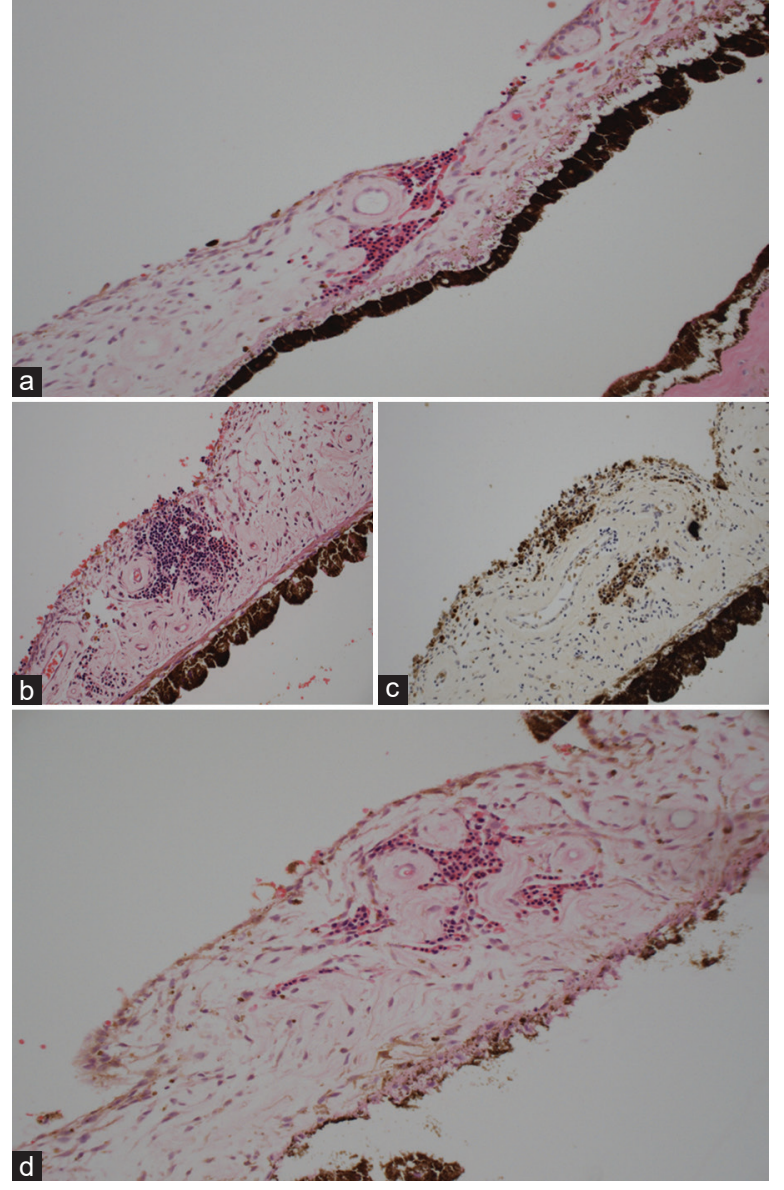

Figure 1: (a) Iris of Patient 1 showing a central cluster of abnormal cells $(\mathrm{H}$ and $\mathrm{E}, \times 20)$. Majority of the cells have dense eosinophilic cytoplasm and rounded nuclei consistent with erythroblasts. (b) Iris of Patient 2 showing clusters of mixed cells including erythroblasts $(\mathrm{H}$ and $\mathrm{E}, \times 20)($ positive with glycophorin A immunohistochemistry, c) and other primitive cells with little cytoplasm (positive with CD117 immunohistochemistry, not shown). (d) Iris stroma of Patient 3 showing clusters of erythroblasts $(H$ and $E, \times 20)$

malignancy. To date, all patients are alive and none had developed metastatic disease.

\section{Discussion}

In our series, the histopathological examination of the three enucleated globes confirmed the diagnosis of $\mathrm{CM}$ and revealed the unusual finding of EMH in the iris. EMH occurs usually in patients with loss of marrow hematopoietic function ${ }^{[8]}$ in the liver, the spleen, or the lymph nodes; other less frequently involved sites include skin, pleura, peritoneum, lung, and para-spinal and epidural spaces. ${ }^{[12]}$

The eye is an uncommon site for hematopoietic elements other than in the context of bone metaplasia in phthisic eyes. EMH has been described in the lacrimal gland, the lacrimal drainage system, and the conjunctiva of patients with systemic myelofibrosis, and the authors suggested that it may be the result of insufficient control of the systemic disease ${ }^{[8,13]}$ Shinde et al. reported EMH in the lacrimal gland in association with 
chronic myeloid leukemia. ${ }^{[13]}$ In contrast to the above, our patients were not affected by blood dyscrasias. Reese and Blodi reported choroidal hematopoiesis in $15 \%$ of their cohort of full-term and premature newborns. ${ }^{[14]}$ In adults, choroidal hematopoiesis was observed after penetrating eye injuries and in association with intraocular B-cell lymphoma. ${ }^{[15,16]}$ The only report of EMH in the iris was a young diabetic patient who underwent vitrectomy and enucleation for neovascular glaucoma complicating central retinal vein occlusion. ${ }^{[17]}$

\section{Conclusion}

This is the first series of CM associated with EMH in the iris. Previous cases of intraocular involvement by EMH were related to systemic myelofibrosis, chronic myeloid leukemia, prematurity, penetrating eye injury, and ocular ischemia. The significance of this finding remains uncertain, but it may be related to the production of inflammatory cytokines stimulating a shift of stem cells to the hematopoietic line. All our patients had previous ocular surgery before enucleation; therefore, possible pathogenic mechanisms include inflammation, ischemia, and production of tumor-related growth factors. Further studies are required to understand whether EMH in UM is related to the primary disease or is secondary to its treatment.

\section{Declaration of patient consent}

The authors certify that they have obtained all appropriate patient consent forms. In the form the patient(s) has/have given his/her/their consent for his/her/their images and other clinical information to be reported in the journal. The patients understand that their names and initials will not be published and due efforts will be made to conceal their identity, but anonymity cannot be guaranteed.

\section{Financial support and sponsorship \\ Nil.}

\section{Conflicts of interest}

There are no conflicts of interest.

\section{RefEREnCES}

1. Singh AD, Turell ME, Topham AK. Uveal melanoma: Trends in incidence, treatment, and survival. Ophthalmology 2011;118:1881-5.

2. Virgili G, Gatta G, Ciccolallo L, Capocaccia R, Biggeri A, Crocetti E, et al. Incidence of uveal melanoma in Europe. Ophthalmology 2007;114:2309-15.

3. McLaughlin CC, Wu XC, Jemal A, Martin HJ, Roche LM, Chen VW. Incidence of noncutaneous melanomas in the U.S. Cancer 2005;103:1000-7.

4. Kaliki S, Shields CL. Uveal melanoma: Relatively rare but deadly cancer. Eye (Lond) 2017;31:241-57.

5. Rodríguez A, Dueñas-Gonzalez A, Delgado-Pelayo S. Clinical presentation and management of uveal melanoma. Mol Clin Oncol 2016;5:675-7.

6. Kujala E, Mäkitie T, Kivelä T. Very long-term prognosis of patients with malignant uveal melanoma. Invest Ophthalmol Vis Sci 2003;44:4651-9.

7. Krantz BA, Dave N, Komatsubara KM, Marr BP, Carvajal RD. Uveal melanoma: Epidemiology, etiology, and treatment of primary disease. Clin Ophthalmol 2017;11:279-89.

8. Ghazi NG, Bowman AM, Shields MD. Bilateral lacrimal system involvement by sclerosing extramedullary hematopoietic tumor. Ophthalmic Plast Reconstr Surg 2006;22:296-8.

9. Lane JE, Walker AN, Kulharya A, Marzec T. Cutaneous sclerosing extramedullary hematopoietic tumor in chronic myelogenous leukemia. J Cutan Pathol 2002;29:608-12.

10. Hill DA, Swanson PE. Myocardial extramedullary hematopoiesis: A clinicopathologic study. Mod Pathol 2000;13:779-87.

11. Wolf BC, Neiman RS. Hypothesis: Splenic filtration and the pathogenesis of extramedullary hematopoiesis in agnogenic myeloid metaplasia. Hematol Pathol 1987;1:77-80.

12. Cuttler N, Heidemann D, Cendrowski C, Armin AR, Folberg R. Extramedullary hematopoiesis of the conjunctiva presenting as active systemic disease in a patient with myelofibrosis. Cornea 2014;33:1352-4.

13. Shinder R, Mirani N, Wu HV, Langer PD. Extramedullary hematopoiesis in the lacrimal gland. Ophthalmic Plast Reconstr Surg 2008;24:48-50.

14. Reese AB, Blodi FC. Hematopoiesis in and around the eye. Am J Ophthalmol 1954;38:214-21.

15. Mudhar HS, Ford AL, Ebrahimi KB, Farr R, Murray A. Intraocular choroidal extramedullary haematopoiesis. Histopathology 2005;46:694-6.

16. Sharpe RW, Bethel KJ, Keefe KS, Hall FW, Levine EM, Regillo CD. Choroidal hematopoiesis in an adult. Arch Ophthalmol 1996;114:1421-2.

17. Wolter JR, Schnitzer B, Wieringa A. Ectopic hematopoiesis in the human iris. Int Ophthalmol 1991;15:267-9. 